DOI: https://doi.org/10.14311/TPFM.2021.001

\title{
NOTE ON THE MATHEMATICAL ANALYSIS OF THE MOTION OF A RIGID BODY IN A GENERALIZED INCOMPRESSIBLE NAVIER - STOKES FLUID
}

\author{
Hind Al Baba ${ }^{1}$, Amrita Ghosh ${ }^{2,3}$, Boris Muha ${ }^{4}$,Šárka Nečasová ${ }^{2}$ \\ ${ }^{1}$ Laboratoire de Mathématiques et Applications, Lebanese University, Beirut, Lebanon, \\ 2 Institute of Mathematics of the Academy of Sciences of the Czech Republic, \\ Žitná 25, 11567 Praha, Czech Republic, \\ 3 Institute for Applied Mathematics, University of Bonn, \\ Endenicher Allee 60, 53115 Bonn, Germany, \\ ${ }^{4}$ Department of Mathematics, Faculty of Science, University of Zagreb, Croatia
}

\begin{abstract}
We deal with a fluid-structure interaction problem: a motion of the rigid body inside a bounded domain filled by a fluid. We consider a viscous incompressible fluid described by the generalized incompressible Navier-Stokes equations which include cases of Newtonian and non-Newtonian fluids. The fluid and the rigid body are coupled via the Navier slip boundary conditions and the motion of the solid is governed by Newton's laws. We also investigate the case of the nonlinear slip condition.The main results assert the existence of strong solutions, in an $L^{p}-L^{q}$ setting.
\end{abstract}

Keywords: Navier-Stokes equations, non-Newtonian fluid, Navier slip condition, strong solution

\section{Introduction}

We study a fluid-rigid body interaction problem in $\mathbb{R}^{3}$. The aim of this article is to develop an $L^{p}$-theory for strong solutions of the coupled system, for both Newtonian and non-Newtonian fluids with the moving rigid body.

We begin with describing the model. We study a system of equations modelling the interaction between a fluid flow satisfying the incompressible generalized Navier-Stokes equations and a rigid body satisfying the conservation of linear and angular momentum. The rigid body moves inside the fluid and at time $t \geq 0$, occupies a bounded domain $\Omega_{S}(t)$, while the fluid fills a bounded domain $\Omega_{F}(t)$. The common boundary of $\Omega_{F}(t)$ and $\Omega_{S}(t)$ is denoted by $\partial \Omega_{S}(t)$. Note that $\Omega_{S}(0) \cup \Omega_{F}(0) \cup \partial \Omega_{S}(0)=\Omega_{S}(t) \cup \Omega_{F}(t) \cup \partial \Omega_{S}(t)=: \Omega \subsetneq \mathbb{R}^{3} ; \quad t \geq 0$ and $\partial \Omega_{F}(t)=\partial \Omega \cup \partial \Omega_{S}(t)$.

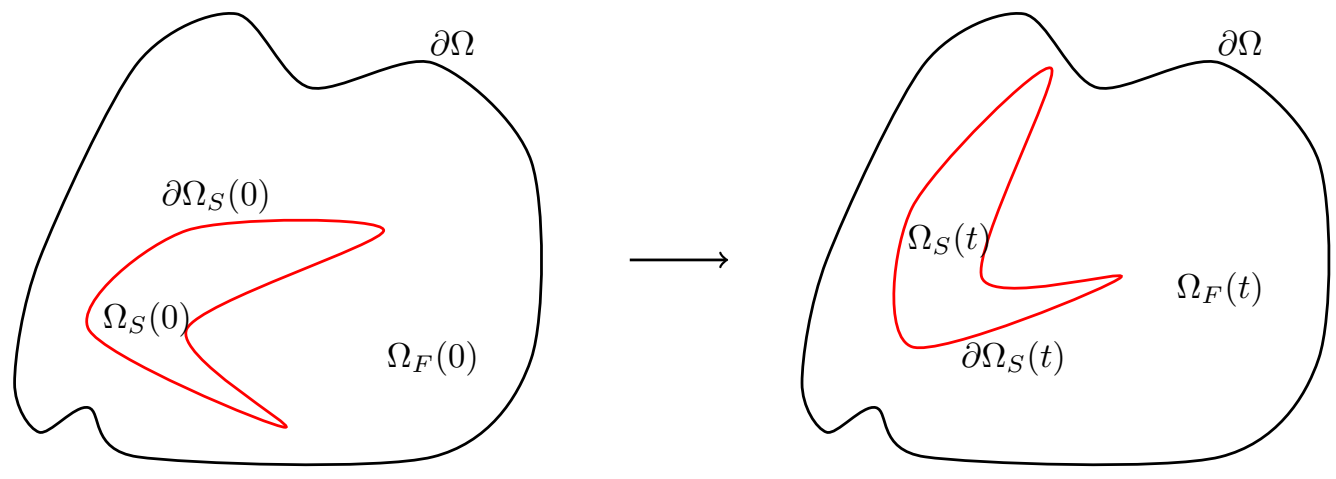

Figure 1: Domain

By choosing a frame of coordinates whose origin initially coincides with the centre of mass of the rigid body, the domain $\Omega_{S}(t)$ at any instant $t$ can be given by

$$
\Omega_{S}(t)=\left\{\boldsymbol{h}(t)+Q(t) \boldsymbol{y}: \boldsymbol{y} \in \Omega_{S}(0)\right\}
$$


where $\boldsymbol{h}(t)$ is the centre of mass of the rigid body at time $t$ and $Q(t)$ is a rotation matrix associated to the angular velocity $\boldsymbol{\omega}(t)$ of the rigid body. The matrix $Q(t)$ is the solution of the initial value problem

$$
\begin{aligned}
\dot{Q}(t) Q^{T}(t) \boldsymbol{y} & =\boldsymbol{\omega}(t) \times \boldsymbol{y} \quad \forall \boldsymbol{y} \in \mathbb{R}^{3} \\
Q(0) & =I_{3} .
\end{aligned}
$$

Here $A^{T}$ denotes the transpose matrix of $A$ and $I_{3}$ is the $3 \times 3$ identity matrix. The system of equations is given by:

$$
\left\{\begin{array}{rlrl}
\partial_{t} \boldsymbol{u}+\left(\boldsymbol{u} \cdot \nabla_{x}\right) \boldsymbol{u} & =\operatorname{div} T(\boldsymbol{u}, \pi) & & \text { in } \Omega_{F}(t) \times(0, T), \\
\operatorname{div} \boldsymbol{u} & =0 & & \text { in } \Omega_{F}(t) \times(0, T), \\
\boldsymbol{u} & =\mathbf{0} & & \text { on } \partial \Omega \times(0, T), \\
\boldsymbol{u} \cdot \boldsymbol{n} & =\boldsymbol{u}_{S} \cdot \boldsymbol{n} & & \text { on } \partial \Omega_{S}(t) \times(0, T), \\
{[T(\boldsymbol{u}, \pi) \boldsymbol{n}]_{\boldsymbol{\tau}}+\alpha \boldsymbol{u}_{\boldsymbol{\tau}}} & =\alpha \boldsymbol{u}_{S \boldsymbol{\tau}} & & \text { on } \partial \Omega_{S}(t) \times(0, T), \\
m \boldsymbol{l}^{\prime}(t) & =-\int_{\partial \Omega_{S}(t)} T(\boldsymbol{u}, \pi) \boldsymbol{n}, & & t \in(0, T), \\
(J \boldsymbol{\omega})^{\prime}(t) & =-\int_{\partial \Omega_{S}(t)}(\boldsymbol{x}-\boldsymbol{h}(t)) \times T(\boldsymbol{u}, \pi) \boldsymbol{n}, & & t \in(0, T), \\
\boldsymbol{u}(0) & =\boldsymbol{u}_{0} & & \text { in } \Omega_{F}(0), \\
\boldsymbol{l}(0)=\boldsymbol{l}_{0}, \quad \boldsymbol{\omega}(0)=\boldsymbol{\omega}_{0}(0) & &
\end{array}\right.
$$

where $\boldsymbol{u}$ and $\pi$ denote the velocity field and pressure of the fluid respectively, $T(\boldsymbol{u}, \pi):=\mu\left(|\mathbb{D} \boldsymbol{u}|^{2}\right) \mathbb{D} \boldsymbol{u}-$ $\pi I_{3}$ is the Cauchy stress tensor with the viscosity function $\mu \in C^{1,1}\left(\mathbb{R}^{+} ; \mathbb{R}\right)$ satisfying the following assumptions

$$
\mu(s)>0 \text { and } \mu(s)+2 s \mu^{\prime}(s)>0 \text { for all } s \geq 0
$$

and $\mathbb{D} \boldsymbol{u}:=\frac{1}{2}\left(\nabla \boldsymbol{u}+\nabla \boldsymbol{u}^{T}\right)$ i.e., $(\mathbb{D} \boldsymbol{u})_{i j}:=\mathbb{D}_{i j} \boldsymbol{u}:=\frac{1}{2}\left(\partial_{i} u_{j}+\partial_{j} u_{i}\right)$ denotes the symmetrical part of the Cauchy stress tensor with $|\mathbb{D} \boldsymbol{u}|^{2}=\sum_{i, j=1}^{3}\left(\mathbb{D}_{i j} \boldsymbol{u}\right)^{2}$ is the Hilbert-Schmidt norm. The friction coefficient $\alpha(\boldsymbol{x}) \geq 0$ is a given function and $\boldsymbol{n}(\boldsymbol{x}, t)$ denotes the unit outward normal vector with respect to the domain $\Omega_{F}(t)$. The subscript $(\cdot)_{\tau}$ denotes the tangential component of a vector i.e. $\boldsymbol{v}_{\boldsymbol{\tau}}=\boldsymbol{v}-(\boldsymbol{v} \cdot \boldsymbol{n}) \boldsymbol{n}$. The constant $m>0$ is the mass of the rigid body and $J(t)$ is its inertia tensor, given by

$$
J(t) a \cdot b=\int_{\Omega_{S}(0)} \rho_{S}(a \times(\boldsymbol{x}-\boldsymbol{h}(t))) \cdot(b \times(\boldsymbol{x}-\boldsymbol{h}(t))) \quad \forall a, b \in \mathbb{R}^{3}
$$

where $\rho_{S}>0$ is the density of the body. Finally, $\boldsymbol{l}(t):=\boldsymbol{h}^{\prime}(t)$ denotes the translational velocity such that $\boldsymbol{u}_{S}(\boldsymbol{x}, t):=\boldsymbol{l}(t)+(\boldsymbol{x}-\boldsymbol{h}(t)) \times \boldsymbol{\omega}(t)$ is the velocity of the rigid body.

Remark 1.1 Let us remark that our stress tensor includes a model of the power-law type fluids, namely,

$$
\mu\left(|\mathbb{D} \boldsymbol{u}|^{2}\right)=\mu_{0}\left(1+|\mathbb{D} \boldsymbol{u}|^{2}\right)^{\frac{d-2}{2}}, \quad \mu\left(|\mathbb{D} \boldsymbol{u}|^{2}\right)=\mu_{0}|\mathbb{D} \boldsymbol{u}|^{d-2} \quad \text { for } \quad \mu_{0} \in(0, \infty), d \in(1, \infty) .
$$

Remark 1.2 (1) The case $d=2$ corresponds to the classical Newtonian fluids i.e. the case of a constant viscosity and we denote the stress tensor by $\sigma(\boldsymbol{u}, \pi)$ given by $\sigma(\boldsymbol{u}, \pi):=\mu_{0} \mathbb{D} \boldsymbol{u}-\pi I_{3}$.

(2) The case $d \in(1,2)$ corresponds to the shear-thinning fluids, which include many important materials of interest (e.g. can be applied for modelling of blood).

(3) The case $d \in(2, \infty)$ corresponds to shear-thickening fluids.

For further discussions on the related non-Newtonian fluids, we refer e.g. [3], [10].

Concerning the study of the fluid-rigid body interaction system involving non-Newtonian fluids, there are not many works done so far. The authors in [7] provide the $L^{p}$-theory for local, strong 
solutions in the case of the Dirichlet coupling condition (no-slip) at the fluid-solid interface and for $\Omega=\mathbb{R}^{3}$. Furthermore in [6], the authors have considered a similar system with only power-law type fluid and with the Dirichlet boundary condition $(\alpha=\infty)$ and establish the existence of a global in time, weak solution. The existence of a weak solution of the Bingham type of fluid with motion of a rigid body, we mention the work in [12].

In the present work, we consider a non-standard boundary condition, known as the Navier slip boundary condition at the fluid-solid interface. This condition state that, apart from being the physical domain impermeable, it allows the fluid to slip over the solid boundary, rather than sticking to it. More precisely, the tangential component of the fluid velocity at the interface need not be equal to the tangential component of the velocity of the solid boundary, and this difference is proportional to the stress exerted by the fluid on the boundary. The proportionality constant is called the friction coefficient (inverse of the slip length). In this article, we focus on the case with the slip condition on the boundary of the rigid body $\partial \Omega_{S}$ and the Dirichlet boundary condition on the outer boundary $\partial \Omega$. Such type of problem is called as mixed case and firstly was investigated in [8]. Several other results for the mixed case can be found in e.g. [4, 1, 5]. All the above-mentioned results are in $L^{2}$-setting, for the Newtonian fluids and the friction coefficient $\alpha$ is assumed either 0 or a constant.

Further, based on molecular dynamics simulations, Thompson and Trojan proposed a slip boundary condition which is highly non-linear [15], even though the fluid is still considered to be Newtonian (see also [11]). This universal condition may determine the degree of slip at a fluidsolid interface as the interfacial parameters and the shear rate vary. But this non-linear boundary condition seems out of reach with our present mathematical technique. However, we may treat the non-linearity discussed by Lewandowski et al. [9] in the context of turbulence model

$$
[\sigma(\boldsymbol{u}, \pi) \boldsymbol{n}]_{\boldsymbol{\tau}}+\alpha|\boldsymbol{u}| \boldsymbol{u}_{\boldsymbol{\tau}}=\mathbf{0}
$$

which is not much different from its linear counterpart, at least concerning the qualitative analysis.

We begin with considering the Newtonian case. The domain $\Omega_{F}(t) \times(0, t)$ depends on the motion of the rigid body, making it a moving boundary problem where the domain is also an unknown a priori. Hence, the natural method is first to transform the system (1.1) to a fixed domain and solve the problem there. We apply the transformation introduced by Tucsnak, Cumsille and Takahashi (cf. $[13,14])$ : a local change of variables which act in a bounded neighbourhood of the body, i.e. it coincides with $Q(t) \boldsymbol{y}+\boldsymbol{h}(t)$ in a neighbourhood of the rigid body and is equal to the identity far from the rigid body. This transformation preserves the solenoidal condition of the fluid velocity and do not change the regularity of the solutions, although the rigid body equations change and become non-linear. Moreover, we use the rotation matrix $Q$ instead of the Jacobian matrix $J_{Y}$ in the change of variables (for details, see [2, Appendix]). Although our transformation does not preserve the divergence free condition as compared to the approach involving $J_{Y}$ used in [13], it makes the computation and corresponding estimates on the non-linear terms, appeared from the change of variables, much easier, since all the derivatives of $Q$ with respect to $\boldsymbol{x}$ can be avoided. After the change of variables, the strategy is based on the maximal regularity property of the linearized system. We extend the maximal regularity result for the Stokes problem to the coupled fluid-rigid body system. At this step, we write the full system in terms of $\mathbb{P} \boldsymbol{u}, \boldsymbol{l}, \boldsymbol{\omega}$ only, where $\mathbb{P}$ is the Helmholtz projection. This helps us to achieve further the exponential stability of the system in the Newtonian case. We finally rewrite the full non-linear transformed problem as a fixed point problem and deduce several estimates on the coordinate transform and on the extra terms appearing from the transformed system which make the fixed point mapping contractive, provided the given data is small. This gives the existence of a unique strong solution in $L^{p}$-spaces of the full fluid-rigid body system.

Also note that the approach in [7] is different from ours. In [7], the fluid and solid equations are treated separately and maximal $L^{p}-L^{q}$ property for the linear fluid-structure system is obtained using some fixed point argument. In our approach, we solve the fluid and structure equations simultaneously. In the study of fluid-structure interactions, this method is known as monolithic approach. The main advantage of such approach is that by studying the resolvent of the linear fluid-structure operator, we can conclude that this operator generates a continuous semigroup of negative type. This allows us to obtain maximal $L^{p}-L^{q}$ regularity for the linear coupled problem 
for $t>0$. As a consequence, one further obtains global existence and uniqueness result for the full non-linear system under some smallness assumption of given data.

Next we concentrate on the non-Newtonian case. Using the same transformation as before, we reduce the system on a fixed domain and then linearize the corresponding operator by fixing the coefficients. To prove the maximal regularity of the linearized system, the same approach as in the Newtonian case can not be followed. Because of the complicated structure of the generalized operator, writing the full system in terms of only $\mathbb{P} \boldsymbol{u}, \boldsymbol{l}, \boldsymbol{\omega}$ seems not possible. Thus we follow here the approach used in [7]. Finally, we show that the same analysis can be carried out as well for the non-linear slip condition (1.2).

Remark 1.3 Note that we can also consider the Navier slip condition $\boldsymbol{u} \cdot \boldsymbol{n}=\mathbf{0},[T(\boldsymbol{u}, \pi) \boldsymbol{n}]_{\boldsymbol{\tau}}+$ $\alpha \boldsymbol{u}_{\boldsymbol{\tau}}=0$ on the outer boundary $\partial \Omega$ instead of $(1.1)_{3}$ and study the system. This makes no big difference in the analysis. The change of variables we used here [Appendix, [2]], do not preserve the Navier condition on $\partial \Omega$, although it conserves the slip condition at the fluid-solid interface. Thus the case with slip condition at the outer fluid boundary can be treated with the usual change of variables (cf. [7]).

\section{Main results}

We assume that the rigid body at the initial position does not touch the wall of the fluid domain, i.e.

$$
\operatorname{dist}\left(\Omega_{S}(0), \partial \Omega\right) \geq \beta>0 \text {. }
$$

For reference purpose, we rewrite the generalized system (1.1) in case of Newtonian fluid:

$$
\left\{\begin{array}{rlrl}
\partial_{t} \boldsymbol{u}+\left(\boldsymbol{u} \cdot \nabla_{x}\right) \boldsymbol{u} & =\operatorname{div} \sigma(\boldsymbol{u}, \pi) & & \text { in } \Omega_{F}(t) \times(0, T), \\
\operatorname{div} \boldsymbol{u} & =0 & & \text { in } \Omega_{F}(t) \times(0, T), \\
\boldsymbol{u} & =\mathbf{0} & & \text { on } \partial \Omega \times(0, T), \\
\boldsymbol{u} \cdot \boldsymbol{n} & =\boldsymbol{u}_{S} \cdot \boldsymbol{n} & & \text { on } \partial \Omega_{S}(t) \times(0, T), \\
2[(\mathbb{D} \boldsymbol{u}) \boldsymbol{n}]_{\boldsymbol{\tau}}+\alpha \boldsymbol{u}_{\boldsymbol{\tau}} & =\alpha \boldsymbol{u}_{S \boldsymbol{\tau}} & & \text { on } \partial \Omega_{S}(t) \times(0, T), \\
m \boldsymbol{l}^{\prime}(t) & =-\int_{\partial \Omega_{S}(t)} \sigma(\boldsymbol{u}, \pi) \boldsymbol{n}, & & t \in(0, T), \\
(J \boldsymbol{\omega})^{\prime}(t) & =-\int_{\partial \Omega_{S}(t)}(\boldsymbol{x}-\boldsymbol{h}(t)) \times \sigma(\boldsymbol{u}, \pi) \boldsymbol{n}, & & t \in(0, T), \\
\boldsymbol{u}(0) & =\boldsymbol{u}_{0} & & \text { in } \Omega_{F}(0), \\
\boldsymbol{l}(0)=\boldsymbol{l}_{0}, \quad \boldsymbol{\omega}(0)=\boldsymbol{\omega}_{0} . &
\end{array}\right.
$$

We use the following function spaces. For a domain $D \in \mathbb{R}^{3}$, the Sobolev spaces are denoted by $W^{m, q}(D)$. For every $0<s<m, m \in \mathbb{N}$ and $1 \leq q<\infty, 1 \leq p \leq \infty$, we denote the Besov spaces by $B_{q, p}^{s}(D)$ which can be defined (equivalently) by real interpolation of Sobolev spaces (cf. [16, Section 1.6.4, page 39])

$$
B_{q, p}^{s}(D):=\left(L^{q}(D), W^{m, q}(D)\right)_{s / m, p} .
$$

We also introduce the following notation, the subscript $\sigma$ denotes the divergence free condition in the domain and the subscript $\boldsymbol{\tau}$ over a space denotes the zero normal component on the boundary. For example, we write:

$$
\boldsymbol{L}_{\sigma, \boldsymbol{\tau}}^{q}(D):=\left\{\boldsymbol{v} \in \boldsymbol{L}^{q}(D): \operatorname{div} \boldsymbol{v}=0 \text { in } D, \boldsymbol{v} \cdot \boldsymbol{n}=0 \text { on } \partial D\right\} .
$$

Let the Stokes operator with Navier boundary conditions on $\boldsymbol{L}_{\sigma, \boldsymbol{\tau}}^{q}\left(\Omega_{F}(0)\right)$ be defined as,

$$
\left\{\begin{array}{l}
\mathcal{D}\left(A_{q}\right):=\left\{\boldsymbol{u} \in \boldsymbol{W}_{\sigma, \boldsymbol{\tau}}^{2, q}\left(\Omega_{F}(0)\right): \boldsymbol{u}=\mathbf{0} \text { on } \partial \Omega, 2[(\mathbb{D} \boldsymbol{u}) \boldsymbol{n}]_{\boldsymbol{\tau}}+\alpha \boldsymbol{u}_{\boldsymbol{\tau}}=\mathbf{0} \text { on } \partial \Omega_{S}(0)\right\} \\
A_{q} \boldsymbol{u}=\mathbb{P} \Delta \boldsymbol{u} \quad \text { for all } \boldsymbol{u} \in \mathcal{D}\left(A_{q}\right)
\end{array}\right.
$$


where $\alpha$ is such that

$$
\alpha \in \begin{cases}W^{1-\frac{1}{\frac{3}{2}+\varepsilon}, \frac{3}{2}+\varepsilon}\left(\partial \Omega_{F}(0)\right) & \text { if } 1<p \leq \frac{3}{2} \\ W^{1-\frac{1}{p}, p}\left(\partial \Omega_{F}(0)\right) & \text { if } p>\frac{3}{2}\end{cases}
$$

with $\varepsilon>0$ arbitrarily small and $\mathbb{P}$ is the Helmholtz projection

$$
\mathbb{P}: \mathbf{L}^{q}\left(\Omega_{F}(0)\right) \rightarrow \mathbf{L}_{\sigma, \boldsymbol{\tau}}^{q}\left(\Omega_{F}(0)\right)
$$

i.e. for $\boldsymbol{\varphi} \in \mathbf{L}^{q}\left(\Omega_{F}(0)\right), \mathbb{P} \boldsymbol{\varphi}=\boldsymbol{\varphi}-\nabla p$ for some $p \in W^{1, q}\left(\Omega_{F}(0)\right)$ which satisfies

$$
\begin{cases}\operatorname{div}(\nabla p-\boldsymbol{\varphi})=0 & \text { in } \Omega_{F}(0) \\ (\nabla p-\boldsymbol{\varphi}) \cdot \boldsymbol{n}=0 & \text { on } \partial \Omega_{F}(0) .\end{cases}
$$

Also we say that $\boldsymbol{u}_{0} \in B_{q, p}^{2-2 / p}\left(\Omega_{F}(0)\right)$ satisfies the compatibility condition if

$$
\begin{aligned}
& \begin{array}{l}
\boldsymbol{u}_{0}-\boldsymbol{v}_{0} \in\left(\boldsymbol{L}_{\sigma, \boldsymbol{\tau}}^{q}\left(\Omega_{F}(0)\right), \mathcal{D}\left(A_{q}\right)\right)_{1-\frac{1}{p}, p} \quad \text { for some } \boldsymbol{v}_{0} \in C^{2}\left(\Omega_{F}(0)\right) \text { satisfying } \\
\operatorname{div} \boldsymbol{v}_{0}=0 \text { in } \Omega_{F}(0), \\
\quad \boldsymbol{v}_{0} \cdot \boldsymbol{n}=\left(\boldsymbol{l}_{0}+\left(\boldsymbol{\omega}_{0} \times y\right)\right) \cdot \boldsymbol{n} \text { on } \partial \Omega_{S}(0), \quad \boldsymbol{v}_{0}=\mathbf{0} \text { on } \partial \Omega, \\
\text { and if } p>3, \quad 2\left[\left(\mathbb{D} \boldsymbol{v}_{0}\right) \boldsymbol{n}\right]_{\boldsymbol{\tau}}+\alpha \boldsymbol{v}_{0 \boldsymbol{\tau}}=\alpha\left(\boldsymbol{l}_{0}+\boldsymbol{\omega}_{0} \times \boldsymbol{y}\right)_{\boldsymbol{\tau}} \text { on } \partial \Omega_{S}(0) .
\end{array}
\end{aligned}
$$

We can now state our main results on the existence of a unique, global in time, strong solutions for the Newtonian and the generalized Newtonian system (2.1) and (1.1) under the smallness assumption on data. For proofs, we refer to [2].

Theorem 2.1 Let $\Omega_{F}(0)$ be a bounded domain of class $\mathcal{C}^{2,1}, p, q \in(1, \infty)$ satisfy the condition $\frac{1}{p}+\frac{3}{2 q} \leq \frac{3}{2}$ and $\alpha \geq 0$ be as in (2.2). Let $\eta \in\left(0, \eta_{0}\right)$ where $\eta_{0}$ is some constant. Then there exist two constants $\delta_{0}>0$ and $C>0$, depending only on $p, q, \eta$ and $\Omega_{F}(0)$ such that for all $\delta \in\left(0, \delta_{0}\right)$ and for all $\left(\boldsymbol{u}_{0}, \boldsymbol{l}_{0}, \boldsymbol{\omega}_{0}\right) \in B_{q, p}^{2(1-1 / p)}\left(\Omega_{F}(0)\right) \times \mathbb{R}^{3} \times \mathbb{R}^{3}$ satisfying the compatibility conditions (2.3) and

$$
\left\|\boldsymbol{u}_{0}\right\|_{B_{q, p}^{2(1-1 / p)}\left(\Omega_{F}(0)\right)}+\left\|\boldsymbol{l}_{0}\right\|_{\mathbb{R}^{3}}+\left\|\boldsymbol{\omega}_{0}\right\|_{\mathbb{R}^{3}} \leq \delta
$$

the system (2.1) possesses a unique global strong solution $(\boldsymbol{u}, \pi, \boldsymbol{l}, \boldsymbol{\omega})$ in the class of functions satisfying

$$
\begin{aligned}
& \left\|e^{\eta(\cdot)} \boldsymbol{u}\right\|_{L^{p}\left(0, \infty ; \boldsymbol{W}^{2, q}\left(\Omega_{F}(\cdot)\right)\right)}+\left\|e^{\eta(\cdot)} \boldsymbol{u}\right\|_{W^{1, p}\left(0, \infty ; \boldsymbol{L}^{q}\left(\Omega_{F}(\cdot)\right)\right)}+\left\|e^{\eta(\cdot)} \boldsymbol{u}\right\|_{L^{\infty}\left(0, \infty ; B_{q, p}^{2(1-1 / p)}\left(\Omega_{F}(\cdot)\right)\right)} \\
+ & \left\|e^{\eta(\cdot)} \pi\right\|_{L^{p}\left(0, \infty ; W^{1, q}\left(\Omega_{F}(\cdot)\right)\right)}+\left\|e^{\eta(\cdot)} \boldsymbol{l}\right\|_{W^{1, p}\left(0, \infty ; \mathbb{R}^{3}\right)}+\left\|e^{\eta(\cdot)} \boldsymbol{\omega}\right\|_{W^{1, p}\left(0, \infty ; \mathbb{R}^{3}\right)} \leq C \delta .
\end{aligned}
$$

Moreover, $\operatorname{dist}\left(\Omega_{S}(t), \partial(\Omega) \geq \beta / 2\right.$ for all $t \in[0, \infty)$.

In particular, we have,

$$
\|\boldsymbol{u}(\cdot, t)\|_{B_{q, p}^{2(1-1 / p)}\left(\Omega_{F}(t)\right)}+\|\boldsymbol{l}(t)\|_{\mathbb{R}^{3}}+\|\boldsymbol{\omega}(t)\|_{\mathbb{R}^{3}} \leq C \delta e^{-\eta t} .
$$

Remark 2.2 If we consider the slip condition on $\partial \Omega \times(0, T)$ in $(2.1)_{3}$ instead of the Dirichlet condition, then we obtain the same result as above, with suitable compatibility condition for the initial data.

Theorem 2.3 Let $p>5, \Omega_{F}(0)$ be a bounded domain of class $\mathcal{C}^{2,1}$ and $\alpha \geq 0$ satisfies (2.2). Then there exists a constant $\delta_{0}>0$ depending only on $p$ and $\Omega_{F}(0)$ such that for all $\delta \in\left(0, \delta_{0}\right)$ and for all $\left(\boldsymbol{u}_{0}, \boldsymbol{l}_{0}, \boldsymbol{\omega}_{0}\right) \in W^{2-2 / p, p}\left(\Omega_{F}(0)\right) \times \mathbb{R}^{3} \times \mathbb{R}^{3}$ satisfying the compatibility conditions (2.3) and

$$
\left\|\boldsymbol{u}_{0}\right\|_{W^{2-2 / p, p}\left(\Omega_{F}(0)\right)}+\left\|\boldsymbol{l}_{0}\right\|_{\mathbb{R}^{3}}+\left\|\boldsymbol{\omega}_{0}\right\|_{\mathbb{R}^{3}} \leq \delta,
$$

the problem (1.1) admits a unique strong solution

$$
\begin{aligned}
& \boldsymbol{u} \in L^{p}\left(0, \infty ; \boldsymbol{W}^{2, p}\left(\Omega_{F}(\cdot)\right)\right) \cap W^{1, p}\left(0, \infty ; \boldsymbol{L}^{p}\left(\Omega_{F}(\cdot)\right)\right) \\
& \pi \in L^{p}\left(0, \infty ; W^{1, p}\left(\Omega_{F}(\cdot)\right)\right), \boldsymbol{l} \in W^{1, p}\left(0, \infty ; \mathbb{R}^{3}\right), \boldsymbol{\omega} \in W^{1, p}\left(0, \infty ; \mathbb{R}^{3}\right) .
\end{aligned}
$$


Remark 2.4 Since the generalized stress tensor $T$ includes the Newtonian stress tensor $\sigma$ as the special case, Theorem 2.3 generalizes Theorem 2.1. On the other hand, less restrictive assumptions on $p, q$ are needed in Theorem 2.1, compared to Theorem 2.3.

Our last result concerns the non-linear slip condition (1.2).

Theorem 2.5 Let $p>5, \Omega_{F}(0)$ be a bounded domain of class $\mathcal{C}^{2,1}$ and $\alpha \geq 0$ satisfies (2.2). Then there exists a constant $\delta_{0}>0$ depending only on $p$ and $\Omega_{F}(0)$ such that for all $\delta \in\left(0, \delta_{0}\right)$ and for all $\left(\boldsymbol{u}_{0}, \boldsymbol{l}_{0}, \boldsymbol{\omega}_{0}\right) \in W^{2-2 / p, p}\left(\Omega_{F}(0)\right) \times \mathbb{R}^{3} \times \mathbb{R}^{3}$ satisfying the compatibility conditions (2.3) and

$$
\left\|\boldsymbol{u}_{0}\right\|_{W^{2-2 / p, p}\left(\Omega_{F}(0)\right)}+\left\|\boldsymbol{l}_{0}\right\|_{\mathbb{R}^{3}}+\left\|\boldsymbol{\omega}_{0}\right\|_{\mathbb{R}^{3}} \leq \delta
$$

the problem (1.1) with the boundary conditions $(1.1)_{5}$ replaced by the non-linear slip condition (1.2) admits a unique strong solution

$$
\begin{aligned}
& \boldsymbol{u} \in L^{p}\left(0, \infty ; \boldsymbol{W}^{2, p}\left(\Omega_{F}(\cdot)\right)\right) \cap W^{1, p}\left(0, \infty ; \boldsymbol{L}^{p}\left(\Omega_{F}(\cdot)\right)\right), \\
& \pi \in L^{p}\left(0, \infty ; W^{1, p}\left(\Omega_{F}(\cdot)\right)\right), \boldsymbol{l} \in W^{1, p}\left(0, \infty ; \mathbb{R}^{3}\right), \boldsymbol{\omega} \in W^{1, p}\left(0, \infty ; \mathbb{R}^{3}\right) .
\end{aligned}
$$

\section{Acknowledgements}

The works of Amrita Ghosh and Šárka Nečasová were supported by the Czech Science Foundation under the Grant No. GA19-04243S in the framework of RVO 67985840 and the work of Boris Muha was supported by the Croatian Science Foundation (Hrvatska zaklada za znanost) Grant IP-2018-01-3706.

\section{References}

[1] H. Al Baba, N. V. Chemetov, Š. Nečasová, and B. Muha: Strong solutions in $L^{2}$ framework for fluid-rigid body interaction problem. Mixed case. Topol. Methods Nonlinear Anal., 52(1):337$350,2018$.

[2] H. Al Baba, A. Ghosh, B. Muha, and Š. Nečasová: $l^{p}$ strong solution to fluid-rigid body interaction system with navier slip boundary condition. arXiv:1912.00352.

[3] H. Bellout and F. Bloom: Incompressible bipolar and non-Newtonian viscous fluid flow. Advances in Mathematical Fluid Mechanics. Birkhäuser, Springer, Cham, 2014.

[4] N. V. Chemetov and Š. Nečasová: The motion of the rigid body in the viscous fluid including collisions. Global solvability result. Nonlinear Anal. Real World Appl., 34:416-445, 2017.

[5] N. V. Chemetov, Š. Nečasová, and B. Muha: Weak-strong uniqueness for fluid-rigid body interaction problem with slip boundary condition. J. Math. Phys., 60(1):011505, 13, 2019.

[6] E. Feireisl, M. Hillairet, and Š. Nečasová: On the motion of several rigid bodies in an incompressible non-Newtonian fluid. Nonlinearity, 21(6):1349-1366, 2008.

[7] M. Geissert, K. Götze, and M. Hieber: $L^{p}$-theory for strong solutions to fluid-rigid body interaction in Newtonian and generalized Newtonian fluids. Trans. Amer. Math. Soc., 365(3):1393$1439,2013$.

[8] D. Gérard-Varet, M. Hillairet, and C. Wang: The influence of boundary conditions on the contact problem in a 3d navier-stokes flow. J. Math. Pures Appl., 9(1):1-38, 2015.

[9] R. Lewandowski, B. Pinier, E. Mémin, and P. Chandramouli: Testing a one-closure equation turbulence model in neutral boundary layers. 2018.

[10] J. Málek, J. Nečas, M. Rokyta, and M. Ružička: Weak and measure-valued solutions to evolutionary PDEs. Applied Mathematics and Mathematical Computation. Chapman and Hall, London, 1996. 
[11] M. T. Matthews and J. M. Hill: Newtonian flow with nonlinear navier boundary condition. Acta Mechanica, 191(3):195-217, Jul 2007.

[12] B. Obando and T. Takahashi: Existence of weak solutions for a bingham fluid-rigid body system. Ann. Inst. H. Poincaré Anal. Non Linéaire, 36(5):1281-1309, 2019.

[13] T. Takahashi: Analysis of strong solutions for the equations modeling the motion of a rigidfluid system in a bounded domain. Adv. Differential Equations, 8(12):1499-1532, 2003.

[14] T. Takahashi and M. Tucsnak: Global strong solutions for the two-dimensional motion of an infinite cylinder in a viscous fluid. J. Math. Fluid Mech., 6(1):53-77, 2004.

[15] P. A. Thompson and S. M. Troian: A general boundary condition for liquid flow at solid surfaces. Nature, 389:360-362, 1997.

[16] H. Triebel: Theory of function spaces. II, volume 84 of Monographs in Mathematics. Birkhäuser Verlag, Basel, 1992. 